\title{
Le cartographe maltais Giovanni Miriti
}

\author{
Joseph Schirò
}

Traducteur : Anne Didier

\section{(2) OpenEdition}

Journals

Édition électronique

URL : http://journals.openedition.org/rbnu/697

DOI : $10.4000 /$ rbnu. 697

ISSN : 2679-6104

Éditeur

Bibliothèque nationale et universitaire de Strasbourg

Édition imprimée

Date de publication : 1 novembre 2017

Pagination : 80-83

ISBN : 9782859230678

ISSN : 2109-2761

\section{Référence électronique}

Joseph Schirò, «Le cartographe maltais Giovanni Miriti », La Revue de la BNU [En ligne], 16 | 2017, mis en ligne le 01 juillet 2019, consulté le 30 décembre 2020. URL : http://journals.openedition.org/rbnu/ 697 ; DOI : https://doi.org/10.4000/rbnu.697

\section{cc) (†) ()}

La Revue de la BNU est mise à disposition selon les termes de la Licence Creative Commons Attribution - Pas d'Utilisation Commerciale - Partage dans les Mêmes Conditions 4.0 International. 


\section{OPVSCVLVM

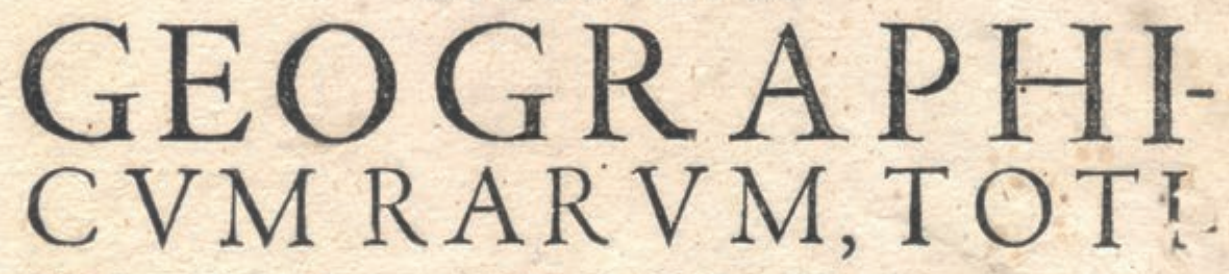 \\ VS EIVS NEGOTII R.ATIO. NEM, MIRA INDVSTRIA ET BREVITATE COMPLECTENS, IAM RECENS EX DIVERSORVM LIBRIS ac chartis, fumma curaac diligentia collectum \& publicatun , per IoAnNem Mroitivm Melitensem, Oru nis Hofpitalis fancti Ioannis Hierofolymitani, Com- mendatorem Alemanni Monafterij, ac domus Ratifponenfis.}

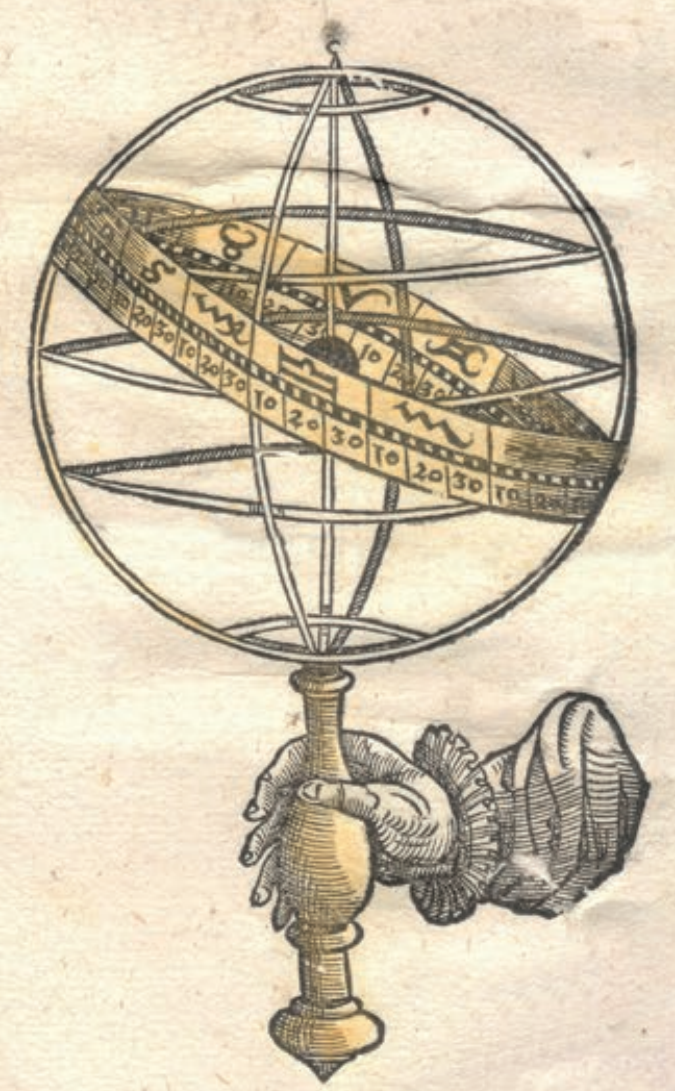

7NGOLSTADII.

Ex Officina Typographica WO LFGANGI $E_{\text {DER I. }}$ A N N O

M. N, LXXXX. 


\section{MALTE \\ LE CARTOGRAPHE MALTAIS GIOVANNI MIRITI

\author{
$\downarrow$ \\ PAR JOSEPH SCHIRÒ \\ (traduction française Anne Didier)
}

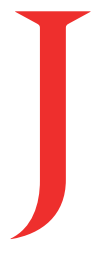

oannes Myritius, dit aussi Giovanni Miriti, savant maltais, a acquis une renommée internationale en 1590 avec la publication de son ouvrage scientifique Opusculum geographicum rarum ${ }^{1}$, imprimé à Ingolstadt, en Allemagne, par Wolfgang Eder. Il y eut deux impressions du livre, une en petit format et l'autre en folio sur grand papier.

Le livre est dédié à son protecteur Frère Philip Reidesel de Kamberg. Il est divisé en deux parties : la première, de la page 1 à 60, a pour titre Prima pars huius opusculi - De principiis astronomiae et geographiae, et traite de questions d'astronomie et de géographie ; la seconde, des pages 61 à 130, décrit le monde (Secunda pars huius opusculi - De particulari orbis descriptione). Le public visé est celui de jeunes étudiants : «[...] quibus adolescentes uti poterunt ad exercendum doctrinam » (p. 54) ${ }^{2}$.

L'ouvrage est illustré de plusieurs gravures sur bois représentant des sphères, des tables astronomiques et des calculs servant à établir latitudes et longitudes, certaines d'entre elles ayant des languettes mobiles et des volvelles ${ }^{3}$. Chaque section est suivie d'appendices détaillés. Le livre contient aussi deux cartes. L'une a pour titre Peloponnesus et montre la côte la plus au sud de la Grèce ainsi que l'extrémité ouest de la Crète. On la trouve page 44. L'autre carte, en regard de la page 61, une carte dépliante du monde intitulée Vniversalis orbis descriptio, porte une bordure décorative en tête, tandis qu'au pied les mots Cogimur e tabula pictos ediscere mundos indiquent à celui qui regarde qu'il peut ainsi connaître les mondes, c'est-à-dire l'ancien et le nouveau. Miriti a placé le nord - Tramontana - en haut de sa carte, dessinée en projection ovale, technique introduite par Francesco Rosselli et Benedetto Bordone au début du $16^{\mathrm{e}}$ siècle. Le monde est entouré de seize têtes de chérubins soufflant le vent, tandis que deux petites cartes des continents sont dessinées aux coins supérieurs de la carte - Mundus novus sive America à gauche et Europa, Africa, Asia à droite. Des citations de Cicéron sont inscrites sur les panneaux des coins inférieurs.

La carte en bois gravé mesure 192 × $322 \mathrm{~mm}$. Le large encadrement décoratif qui l'entoure est agrémenté de rameaux de myrte. On a dit que ceux-ci étaient associés au nom de la famille Miriti qui semble tirer son origine de cette plante, de la famille des myrtacées. En effet, l'encadrement du portrait de Miriti et 
son écusson, reproduits respectivement pages 134 et 135 (voir ill. p. 83), montrent la même feuille de myrte. En tête de ces deux pages sont imprimés les mots Effigies et Insignia autoris (portrait et emblème de l'auteur).

On a écrit que Giovanni Miriti semble avoir été un des derniers géographes qui entreprirent de réconcilier les nouvelles découvertes avec les théories antérieures. Dans la description des territoires, les continents d'Afrique et d'Amérique du Sud sont dessinés assez fidèlement. Cependant Miriti refuse l'idée de la séparation de l'Asie et de l'Amérique du Nord ou de cette dernière et du Groenland. À ses yeux, le tout fait partie de l'" India extra Gangem » (l'Inde au-delà du Gange). Les premières cartes du monde de type Gastaldi, et ce encore en 1560, montrent les deux continents solidement rattachés en un seul territoire. Pour rendre justice à Miriti, nous devons remarquer que la séparation de l'Asie et de l'Amérique, et la reconnaissance de cette dernière comme un continent à part avec tout ce que cela implique d'un point de vue ethnologique, zoologique et botanique, ne concernaient pas seulement des problèmes géographiques, mais aussi de sérieuses questions théologiques que Miriti aurait eu à surmonter avant d'être en mesure d'obtenir une autorisation ecclésiastique pour un livre destiné à l'éducation de la jeunesse catholique.

L'auteur nous montre aussi, aux deux côtés de sa carte du monde, ce qu'on appelle des « climata ». Ceux-ci étaient un moyen de diviser la Terre en zones liées à la durée du jour le plus long d'une zone particulière, mais étant donné qu'au $16^{\mathrm{e}}$ siècle, tous les cartographes réputés avaient rejeté la théorie des climata et avaient déjà commencé à diviser le monde uniquement en longitudes et latitudes, Miriti utilisa les deux systèmes et représenta à la fois les climata et les longitudes et latitudes.

Il naquit à Malte en 1534 et il était très fier du pays de sa naissance. Il se nomme lui-même deux fois maltais dans le livre, d'abord dans le titre écrit par "Ioannem Myritium Melitensem ", puis de nouveau dans la dédicace à Riedesel signée « Ioannes Myritius Melitensis ». En outre, dans sa brève description de Malte, il se réfère à l'île comme "patria mea dulcissima " (ma très douce patrie, p. 89). Bien qu'il eût vécu le plus souvent à l'étranger, Malte continua à tenir une place privilégiée dans son cœur.
Miriti donne dans son livre une courte description de Malte, dans le chapitre intitulé De Africa et eius partibus qui fait référence à l'Afrique et aux îles adjacentes : " Insulae autem adiacent Africae iuxta continentem hae. Hydras [...] Zerbas [...] Lopadusa [...] Cossiro vulgò Pantallaria, Glauconis insula, et Galos vulgò Gozo [...] » (p. 88). Il y écrit : «Il y a aussi Melite, aujourd'hui connue sous le nom de Malte, célèbre par l'arrivée de saint Paul après son naufrage, mon propre et très aimé pays natal. Après la perte de Rhodes et par l'entremise du grand maître Philippe Villiers de l'IsleAdam, l'empereur Charles octroya l'île aux illustres chevaliers de Jérusalem comme nouveau siège le 4 octobre 1530 [la date est une erreur de l'auteur, la date réelle étant le 23 mars 1530], afin qu'un changement de circonstances ne les privât pas de la mer, et ils continuèrent à défendre très glorieusement leur île contre les attaques des Turcs» (p. 89).

Le volume se termine avec des couplets louangeurs pour Giovanni Miriti et son travail. Le premier fut écrit par un Anglais, docteur en médecine, le professeur Edmund Hollyng, et a pour titre Acclamatio Heroica, ad insignem nobilitate, virtute, et eruditione virum, dominum Ioannem Myritium Melitensem \&c. inscripta ab Edmundo Hollyngo Anglo, Medicinae Doctore, et apud Ingolstadienses Professore. Il est suivi d'une épigramme écrite par Ioannes Ritterius et louant le travail de Miriti (In laudem eiusdem operis geographici...).

Seuls quelques détails de l'éducation de Miriti sont parvenus jusqu'à nous, et stipulent simplement que Miriti était " adorno di belle lettere " (paré de belles lettres). Les parents de Giovanni, probablement une noble famille de Rhodes, qui avaient accompagné les chevaliers hospitaliers de Saint-Jean quand ils furent expulsés de cette première île et s'installèrent dans la seconde, lui enseignèrent les arts libéraux. Il devint chapelain conventuel dans la Langue d'Allemagne (branche germanique) de l'ordre de Saint-Jean. Les services rendus à l'ordre ne l'empêchèrent pas de se consacrer sérieusement à ses études, au bout desquelles il obtint un doctorat en droit. Son sujet de prédilection cependant était les mathématiques, et il devint si compétent en la matière qu'il finit par publier en 1590 l'ouvrage de géographie et d'astronomie déjà mentionné, l’Opusculum. 
Dans les années 1560, Miriti fut très occupé par la rénovation des anciens bâtiments de sa commanderie d'Altmünster qui en avaient bien besoin. Une inscription, qui n'existe apparemment plus, y avait été placée en 1568 pour attester et commémorer l'événement. À cette période, Miriti évita de revenir à Malte. Entre 1562 et 1566, il reçut du grand maître Jean de Valette trois sévères lettres déplorant son absence et son silence. Il vint probablement à Malte quand la restauration fut achevée, ayant reçu permission de retourner dans sa commanderie le 18 juin 1569. Plus tard, dans les années 1580, Miriti se mit lui-même dans de sérieux ennuis quand, suivant les pas de ses prédécesseurs, il engagea la propriété de la commanderie sans autorisation pour lever les fonds nécessaires à sa sauvegarde. Un mandat criminel fut même lancé contre lui pour examen de l'affaire. On n'en connaît pas la suite.

La date et le lieu où mourut Giovanni Miriti sont inconnus. Il était encore en vie en septembre 1593, jouissant de sa commanderie, et ce n'est qu'au début de mars 1595 qu'on parla de lui comme de l'ancien commandeur d'Altmünster, ce qui peut vouloir dire qu'il mourut entre ces deux dates.

Giovanni Miriti a l'honneur d'avoir été le premier auteur maltais à publier un livre.

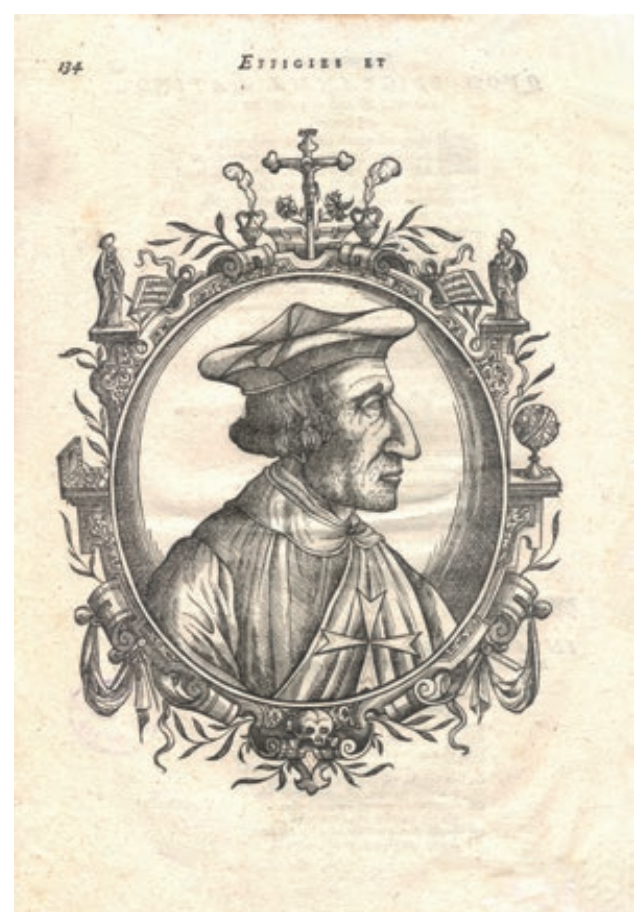

\section{ORIENTATIONS BIBLIOGRAPHIQUES}

- Agius-Vadalà, Maurice, « Giovanni Myriti (1535-1590?) his life and works ", in Proceedings of History Week 1983, Malta, Malta Historical Society, 1984, p. 39-56

- Ganado, Albert, The Early Maltese Cartographers, Malta, Malta Map Society, 2011, p. 24-30

NOTES

1- Le titre complet de l'ouvrage est : Opusculum geographicum rarum, totius eius negotii rationem, mira industria et brevitate complectens, iam recens ex diversorum libris ac chartis, summa cura ac diligentia collectum \& publicatum, per Ioannem Myritium Melitensem.

2- Littéralement : «dont les jeunes gens pourront se servir pour exercer leur savoir ».

3- Les volvelles et les languettes étaient des dispositifs mécaniques dont la fonction dans le livre était de déterminer différentes positions pour un temps donné. Ainsi, dans l'ouvrage de Jacques Bassantin Astronomique discours, imprimé à Lyon en 1557, un diagramme circulaire complexe, avec des parties mobiles, est là « pour trouuer le vray lieu de Venus au Zodiaque ».

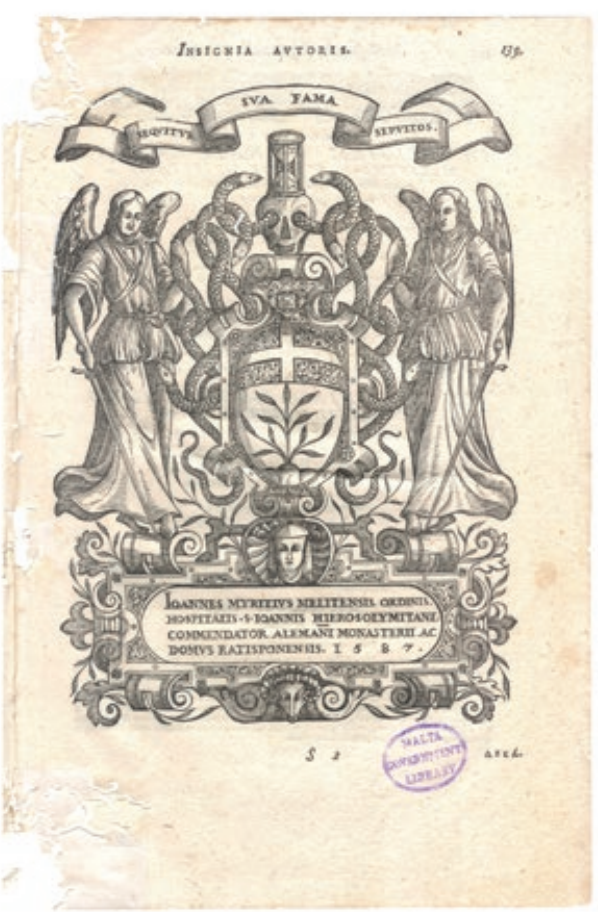

